\title{
New members
}

Anderson, Timothy S, KCOM '79; 10414 Beardslee Blvd, Bothell, WA 98011-3299

Benn, Harvey S, PCOM '74; 1299 Brace Rd, Cherry Hill, NJ 08034-3275

Bennett, Robert H, UMDNJ-SOM '85; 20 Pavilion Rd, Voorhees, NJ 08043

Bickley, Mark S, TCOM '88; 6521 Melwood St, Ft Worth, TX 76112-1784

Brown, Terry M, COMOSU '77; PO Box 699, Judsonia, AR 72081

Caplan, Randy H, UNECOM ' 88 ; 40 Beauvoir Ave, Summit, NJ 07901

Catron, Mark Wesley, UHS/COM '84; 305 E Grand, Jasper, MO 64755

Daniels, Joseph, CCOM '86; 2709 Hospital Blvd, Grand Prairie, TX 75051-1091
David, Henry E, UHS/COM '68; 28 Southwood Dr, Cherry Hill, NJ 08003-2950

DeGennaro, Marianne E, UHS/COM '87; 36 Red Coach Ln, Holmdel, NJ 07733-1137

DelPilar, Arnold, KCOM '89; 54732 Little Flower Trl, Mishawaka, IN 46545-1604

Foster, Theodore F, UHS/COM '60; 7329 Broadway, Kansas City, MO 64114-1357

Frazier, Jay J, KCOM '77; 800 E Pennell, Carl Junction, MO 648349502

Friedman, E Glenn, PCOM '77; 1601 Second Ave, York, PA 17403

Giulitto, Dean R, UOMHS '78; 96b Schofield Circ, Ft Riley, KS 66442-1127

Goykovich, Stephen, WVSOM '80; PO Box 460, Carson City, MI 48811
Hubner, Richard Thomas, TCOM '85; PO Box 639, Springtown, TX 76082-0639

Kardos, Stephen A, KCOM '68; 7 S Arlene Dr, West Long Branch, NJ 07764

Kellum, Daniel H, UHS/COM '73; 7323 Marbach Rd, San Antonio, TX 78227-1905

Kelley, Thomas P, CCOM '73; 20201 S Crawford, Olympia Fields, IL 60461-1010

Kim, James B, NYCOM '87; 30-55 84th St, Jackson Heights, NY 11370

Koerner, Steven T, UHS/COM '89; 1806 Augusta Cir, Mount Laurel, NJ 08054-2759

LaRue, David M, COMOSU '81; 1116 Grand, Carthage, MO 64836-3831

Lorenz, Constance J, TCOM '85; 3750 S University, Ft Worth,

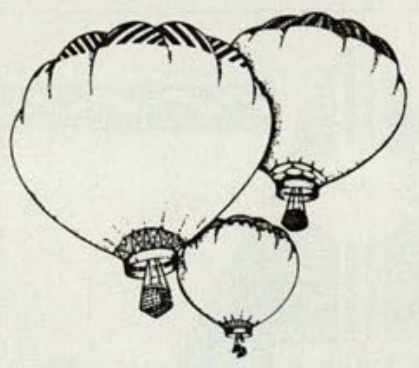

New Mexico Osteopathic Medical Association presents the

\section{1th Annual \\ Hot Air Balloon Fiesta \\ Medical Symposium}

October 3-6, 1991

Holiday Inn Pyramid Hotel

Albuquerque, New Mexico

David C. Leech, DO, Program Chairman

Topics: An OMT workshop, and updates in Internal Medicine, Wilderness Medicine, Sports Medicine, Medical History, and Aerospace Medicine

Anticipating 22 hrs Category 1-A CME Credit, AOA approved.

For registration information, call 505 828-1905, or write

NMOMA

Symposium Information

P O Box 3096

Albuquerque NM 87190-3096 
TX 76109

Lurakis, Michael F, PCOM ' 80 ; Route 50, Mays Landing, NJ 08330-1666

MacMaster, John C, MSUCOM '86; 177 N Barlow Rd, Lincoln, MI 48742

Merrill-Wilson, Patricia, COMP '88; 2688 Gordon Rd, North Pole, AK 99705-6627

Mindlin, Lyle, MSUCOM '79; 6060 Carriage Hill Dr, East Lansing, MI 48823

Miyazaki, William T, COMP '83; 3894 Vistacrest Dr, Reno, NV 89509-7436

Moors, William J, PCOM '70; 525 Rt 73 S Rd 1, Marlton, NJ 08053 9642

Morrell, Rocco L, TCOM '79; 5502 39th St, Groves, TX 77619-2929
Munn, Richard F, UHS/COM '62; 220 Third Ave, Charleston, SC 29403

Napier, James A, UOMHS '74; 12800 Indian Rocks Rd, Largo, FL 34644-2032

Panousieris, Edward, UHS/COM '86; 1143 S Buckner, Dallas, TX 75217

Parker, Charles E, PCOM '68; 168 Business Park Dr, Virginia Beach, VA 23462-6532

Pei, Sun Fai, MSUCOM '83; 1 Fairfield Ln, New York, NY 110401828

Rasor, John B, MSUCOM '85; 5630 Pineridge Ln, Brighton, MI 48116

Riggs, Peyton D, COMP '88; 24214 Jimson PL, Moreno Valley, CA 92388-3547
Rimle, Darlene A, MSUCOM '86; 3301 S Bear, Santa Ana, CA 92704-7240

Romney, Lorenzo M, COMP '86; 1006 S Jackson, Hugoton, KS 67951-0010

Stahr, Richard F, UHS/COM '78; 288 Castille Ln, Westerville, OH 43081-2769

Tatom, Andrew J, PCOM ' $62 ; 160$ Atlantic Ave, Hempstead, NY 11550

Thomas, E David, KCOM '81; 6803 Mayfield Rd, Mayfield Heights, OH 44124-227

Varnado, Stanford M, KCOM '86; 12170 E Kepner Pl, Aurora, CO 80012

Way, Bill V, UHS/COM '75; 7125 Marvin D Love Freeway, Dallas, TX 75237-3111

\section{NEW MEXICO OSTEOPATHIC MEDICAL ASSOCIATION 54TH Annual Convention and Trade Show}

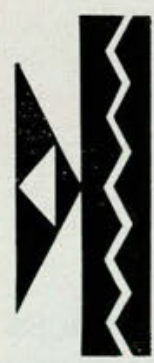

\section{JULY 3-7, 1991}

\section{Holiday Inn Pyramid Hotel Albuquerque, New Mexico}

David C. Leech, DO, Program Chairman

Anticipating 30 hrs Category 1-A CME credit, AOA approved

Topics: The Knee, Office Gynecology, Urology, The Eye, Pediatrics, and Native Amercian Medicine

For registration information, call (505) 828-1905 or write: NMOMA/Convention Information

P.O. Box 3096

Albuquerque, NM 87190-3096 
